Mean jovicentric right ascension

Right ascension of perijove

Right ascension of node on equator

Inclination to equator

Elements referred to the Earth's equator.

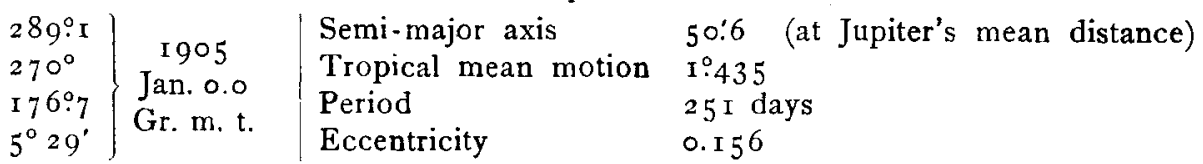

Ephemeris for Greenwich Mean Noon.

\begin{tabular}{|c|c|c|}
\hline 1905 & $p$ & $s$ \\
\hline Aug. 30 & $302^{\circ}$ & $3^{2^{\prime}}$ \\
\hline Sept. 4 & 295 & 37 \\
\hline 9 & 289 & $4 \mathrm{r}$ \\
\hline 14 & 284 & 45 \\
\hline 19 & 279 & 49 \\
\hline 24 & 275 & $5^{2}$ \\
\hline 29 & 272 & 54 \\
\hline Oct. 4 & 268 & 55 \\
\hline 9 & 264 & 55 \\
\hline I 4 & 260 & 55 \\
\hline 19 & 256 & 53 \\
\hline 24 & $25^{1}$ & $5^{I}$ \\
\hline 29 & 246 & $4^{8}$ \\
\hline Nov. 3 & 240 & 44 \\
\hline 8 & 233 & 40 \\
\hline
\end{tabular}

\begin{tabular}{|c|c|c|c|}
\hline \multicolumn{2}{|c|}{ I 905-०6 } & $p$ & $s$ \\
\hline \multirow[t]{4}{*}{ Nov. } & 13 & $225^{\circ}$ & $36^{\circ}$ \\
\hline & 18 & 214 & 33 \\
\hline & 23 & $20 \mathrm{I}$ & 30 \\
\hline & 28 & 186 & 29 \\
\hline \multirow[t]{6}{*}{ Dec. } & 3 & 170 & 30 \\
\hline & 8 & 157 & 32 \\
\hline & 13 & 145 & 35 \\
\hline & 18 & $13^{6}$ & 39 \\
\hline & 23 & I 29 & 43 \\
\hline & 28 & I 23 & 47 \\
\hline \multirow[t]{5}{*}{ Jan. } & 2 & I I 8 & 50 \\
\hline & 7 & I I 4 & 53 \\
\hline & I 2 & I I I & 56 \\
\hline & 17 & 108 & $5^{8}$ \\
\hline & 22 & 105 & 59 \\
\hline
\end{tabular}

\begin{tabular}{|c|c|c|}
\hline I 906 & $p$ & $s$ \\
\hline Jan. 27 & $102^{\circ}$ & $60^{\circ}$ \\
\hline Febr. I & 99 & 60 \\
\hline 6 & 97 & 59 \\
\hline I I & 94 & $5^{8}$ \\
\hline I 6 & 92 & 57 \\
\hline $2 \mathrm{I}$ & 90 & 55 \\
\hline 26 & 88 & 53 \\
\hline Mar. 3 & 85 & 50 \\
\hline 8 & 82 & 47 \\
\hline I 3 & 79 & 43 \\
\hline . 18 & 75 & 39 \\
\hline 23 & 71 & 35 \\
\hline 28 & 65 & $3 r$ \\
\hline
\end{tabular}

Washington, D. C., I g०5 Aug. 5.

VII. Jupitermond.

(Telegramm aus Cambridge, Mass., vom I6. August.)

Tucker telegraphs: Albrecht has observed seventh satellite of Jupiter with Crossley reflector as follows:

$$
\text { r } 905 \text { Aug. } 7.96 \text { Gr. m. t. } \quad \text { P.A. }=289^{\circ} 7 \quad \text { Dist. }=54: 6
$$

The satellite was also observed Aug. 8 and 9 .

Pickering.

Die Beobachtungszeit kann auch Aug. 8.00 gelesen werden; Codewort verstümmelt.

Kiel, 1905 Aug. I 6 .

$K r$.

\title{
Literarische Anzeigen.
}

H. Kayser, Handbuch der Spectroscopie. Dritter Band. Leipzig r905.

Der vorliegende dritte Band des groß angelegten Werkes enthält die Erscheinungen der Absorption, soweit sie nicht, wie die natürlichen organischen Farbstoffe und die mit der Absorption zusammenhängenden Erscheinungen der Dispersion, Fluoreszenz und Phosphoreszenz dem nächsten Bande vorbehalten bleiben. Das dritte Kapitel, enthaltend die Beziehungen zwischen Absorption und Konstitution organischer Körper ist ein Beitrag von Prof. Hartley in Dublin.

F. Bidsch of und A.Vital. Fünfstellige mathematische und astronomische Tafeln. Zum Gebrauche für Mathemathiker, Astronomen, Geographen und Seeleute zusammengestellt und mit Formelsammlungen versehen. Wien und Leipzig r 905 .

Die für astronomische Zwecke bestimmten Tafeln nehmen einen hervorragenden Platz in dieser Tafelsammlung ein. Sowohl die sphärische Astronomie wie die Bestimmung der Bahnen von Planeten und Kometen sind innerhalb der durch die Beschränkung auf fünfstellige Logarithmen gesteckten Grenzen ausreichend berücksichtigt. Die Formelsammlung am Schlusse des Buches wird sich in vieler Beziehung als sehr brauchbar erweisen.

Nover Planet 1905 QX und Planet 1905 QV. Nach einer Mitteilung von Prof. $A$. Berberich vom r4. d. Mts. ist der Planet $1905 \mathrm{QV}$ sehr wahrscheinlich identisch mit $\left(26_{3}\right)$ Dresda, während der für Dresda gehaltene Planet (vergl. Nr. 4039 p. I09 und III) als neu anzusehen und init I905 QX zu bezeichnen ist. $K r$.

Inhalt zu Nr. 4042. B. Viaro. Stelle fra $45^{\circ}$ e $55^{\circ}$ di declinazione boreale. 145. - 7. Palisa. Beobachtungen von kleinen Planeten. I 5 I. L. Terkán. Lichtmessing aller Sterne der Zone $0^{\circ}$ bis - $10^{\circ}$ des südlichen Himmels bis zur GröBenklasse 6.0. Teil I. I53. - F. E. Ross. Elements and ephemeris of Jupiter's sixth satellite. I57. - Pickering. VII. Jupitermond. I59. - Literarische Anzeigen. I59. - Mitteilung uber kleine Planeten. I 59.

Geschlossen 1905 Aug. 2r. Herausgebex: H. Kreutz. Druck von C. Schaidt. Expedition: Kiel, Niemannsweg ro3. 